\title{
COMPUTATIONAL INTELLIGENCE BASED SIMULATED ANNEALING GUIDED KEY GENERATION IN WIRELESS COMMUNICATION (CISAKG)
}

\author{
ARINDAM SARKAR ${ }^{1}$ AND J. K. MANDAL ${ }^{2}$ \\ ${ }^{1}$ Department of Computer Science \& Engineering, University of Kalyani, W.B, India \\ ${ }^{2}$ Department of Computer Science \& Engineering, University of Kalyani, W.B, India
}

\begin{abstract}
In this paper, a Computational Intelligence based Simulated Annealing (SA) guided approach is use to construct the key stream. SA is a randomization technique for solving optimization problems. It is a procedure for finding good quality solutions to a large diversity of combinatorial optimization problems. This technique can assist to stay away from the problem of getting stuck in local optima and to escort towards the globally optimum solution. It is inspired by the annealing procedure in metallurgy. At high temperatures, the molecules of liquid move freely with respect to one another. If the liquid is cooled slowly, thermal mobility is lost. Parametric tests are done and results are compared with some existing classical techniques, which shows comparable results for the proposed system.
\end{abstract}

\section{KEYWORDS}

Simulated Annealing (SA), Key Genaeration, Computational Intelligence..

\section{INTRODUCTION}

In recent times wide ranges of techniques are developed to protect data and information from eavesdroppers [1]-[4]. These algorithms have their merits and shortcomings. For Example in DES, AES [4] algorithms the cipher block length is nonflexible. ANNRPMS [1] and ANNRBLC [2] allow only one cipher block encoding. In NNSKECC algorithm [3] any intermediate blocks throughout its cycle taken as the encrypted block and this number of iterations acts as secret key. In this paper we have proposed a SA based encryption technique for wireless communication.

The organization of this paper is as follows. Section 2 of the paper deals with the proposed SA based key generation technique. Example of the key generation and encryption technique has been discussed in section 3. Results are described in section 4. Conclusions are drawn in section 5 and that of references at end.

\section{The SA BASED Key GeNERATION TeChNiQue}

In SA, the solution starts with a high temperature, and a sequence of trail vectors are generated until inner thermal equilibrium is reached. Once the thermal equilibrium is reached at a particular temperature, the temperature is reduced and a new sequence of moves will start. This process is continued until a sufficiently low temperature is reached, at which no further improvement in the objective function can be achieved. Thus, SA algorithm consists of: configurations, reconfiguration technique, cost function, and cooling schedule. 
The key stream generators considered here are LFSR-based generators. In Key generation process some of the operators are being used in a particular format. The following table illustrated the format of the operator and their corresponding meaning.

Table 1. Operator's format and their meaning

\begin{tabular}{|c|l|l|}
\hline Operator & Format & \multicolumn{1}{c|}{ Meaning } \\
\hline $\mathrm{I}$ & $\mathrm{ab}$ & Bitwise OR \\
\hline$\&$ & $\& \mathrm{ab}$ & Bitwise AND \\
\hline$\wedge$ & $\wedge \mathrm{ab}$ & Bitwise XOR \\
\hline $\mathrm{X}$ & & Character sequence from ' $\mathrm{a}$ '... p' represents the number $0 . .15$ \\
\hline $\mathrm{SR}$ & $\mathrm{SRx}$ & $\begin{array}{l}\text { Shift Register is represents as SR and } \mathrm{x} \text { denotes the feedback } \\
\text { polynomial }\end{array}$ \\
\hline
\end{tabular}

\subsection{Chromosome Representation Scheme}

The population chromosome that represents candidate key stream generators is strings of characters which are expressions represented using prefix notation. These syntactic rules should be preserved during the generation of the initial population, and by the genetic operations. The initial states and feedback functions of the shift registers are represented as strings of the letters 'a'..'p'. These letters represent the numbers $0 . .15$. Thus, each letter is a sequence of four bits. The length of a LFSR is determined by the number of letters which are initially generated randomly. The number of these letters must be even, half of them for the initial state, and the second half for the feedback function. For example, if the number of these letters is eight letters, then four letters are used for the feedback function, thus, the length of LFSR is 16 bits $(4 \times 4)$. Furthermore, the first zeros of the feedback function are ignored. For example, consider the LFSR:"SR abid", ' $i$ ' is the number $8=(1000)_{2}$, then the first three zeros are ignored, and the length of this LFSR will be five bits $(1+4)$. Thus the feedback function will be $(11100)$, or $g(x)=1+x+x^{2}+x^{5}$.

The following are examples of the chromosomes:

Chromosome: SRggbkbecdeh

Chromosome: $\wedge \wedge \& \mid$ SRbpeiSRhoionm $\wedge$ SRlhhk\&SRfmcddiphhcSRcgpjkgSRiechSRkhji

Chromosome: $\wedge$ SRdcaeSRagojdfojfm

Chromosome: |\&SRccga^SReehk\&|SRpfdmingc^SRjeSRjmlidmbeSRhoSRmhofoh

Chromosome: SRlepjgc

\subsection{Construction of Fitness Function}

The fitness value is a measurement of the goodness of the key stream generator, and it is used to control the application of the operations that modify a population. There are a number of metrics used to analyze key stream generators, which are key stream randomness, linear complexity and correlation immunity. Therefore, these metrics should be taken in account in designing key stream generators, and they are in general hard to be achieved. The fitness value is calculated by generating the key stream after executing the program, and then the generated key stream is examined. The fitness function used to evaluate the chromosomes is to calculate at what percentage the chromosome satisfies the desired properties of the stream ciphers. Three factors are considered in the fitness evaluation of the chromosomes which are: 
1. Randomness of the generated key stream.

2. Key stream period length.

3. Chromosome length.

Following equation is used for the evaluation of key stream randomness using the frequency and serial tests, in which, $n w$ is the frequency of $w$ in the generated binary sequence.

This function is derived from the fact that in the random sequence:

1. Probability $\left(\mathrm{n}_{\mathrm{o}}\right)=$ Probability $\left(\mathrm{n}_{1}\right)$, and

2. Probability $\left(\mathrm{n}_{01}\right)=$ Probability $\left(\mathrm{n}_{11}\right)=$ Probability $\left(\mathrm{n}_{10}\right)=$ Probability $\left(\mathrm{n}_{00}\right)$

$$
f_{1}=\left|n_{0}-n_{1}\right|+\left|n_{00}-\frac{S Z}{4}\right|+\left|n_{01}-\frac{S Z}{4}\right|+\left|n_{10}-\frac{S Z}{4}\right|+\left|n_{11}-\frac{S Z}{4}\right|
$$

There is another randomness requirement which is: $\frac{1}{2^{i}} \times n_{r}$ of the runs in the sequence are of length $i$, where $n_{r}$ is the number of runs in the sequence. Thus, we have the following function:

$$
f_{2}=\sum_{i=1}^{M}\left|\left(\frac{1}{2^{i}} \times n_{r}\right)-n_{i}\right|
$$

where $M$ is maximum run length, and $n$ is the desired number of runs of length $i$. Another factor is considered in the evaluation of the fitness value which is the size of the candidate key stream generator (length of the chromosome). Thus, the fitness function used to evaluate the chromosome $\mathrm{x}$ will be as follows, where $w t$ is a constant and size is the key stream period length:

$$
\text { fitness }(x)=\frac{S Z}{1+f_{1}+f_{2}}+\frac{\text { weight }}{\text { length }(x)}
$$

\title{
2.3 Parameters value of the Algorithm
}

The parameters used in this work were set based on the experimental results, the parameter value that show the highest performance was chosen to be used in the implementation of the algorithm. Thus, the genetic operations used to update the population are single point crossover with probability $\mathrm{pc}=1.0$ and mutation with probability $\mathrm{pm}=0.1$. The selection strategy, used to select chromosomes for the genetic operations, is the 2- tournament selection. The old population is completely replaced by the new population which is generated from the old population by applying the genetic operations. Regarding the structure of each chromosome, the maximum chromosome length is 300 characters, and the maximum number of functions (except SR) is ten functions. The probability of the function SR is 0.5 , and all other function are of probability 0.5 . Finally, the maximum LFSR length is 20 bits. The run of GP is stopped after a fixed number of generations. The solution is the best chromosome of the last generation.

\author{
Algorithm Simulated Annealing based Key Stream Generation \\ 1: Input : Length of the key stream \\ 2: Output : Simulated Annealing based key stream \\ Method: \\ 3: Generate the initial population (pop) randomly \\ 4: Evaluate pop \\ 5: temp $\leftarrow 250$. \\ 6: while not Max Number of generations do \\ 7: Generate a new population (popl) by applying crossover and mutation
}


8: Evaluate the fitness of the new generated chromosomes of pop1

9: Calculate the averages of fitness values for pop and pop1, av and avl respectively

10: If $(a v 1>a v)$ then replace the old population by the new one, i.e. pop $\leftarrow$ popl

11: Else

12: Begin

13: $e \leftarrow a v-a v 1$

14: $\mathrm{Pr} \leftarrow$ e/Temp

15: Generate a random number (rnd)

16: If $(\exp (-p r)>r n d)$ then $p o p \leftarrow$ pop1

17: End Else

18: EndIf

19: Temp $\leftarrow$ Temp $* 0.95$

20: end while

21: Return the best chromosome of the last generation

\section{EXAMPLE OF KEY STREAM GENERATION AND SA BASED ENCRYPTION}

Consider Initial population size as 200 and randomly generated each key stream having 128 bits. Then population gets evaluated with the help of fitness function by passes through a number of statistical tests to examine whether the pseudorandom number sequences are sufficiently random or not, which are frequency test, serial test, poker test, auto correlation test and runs test.

1. Frequency Test: It calculates the number of ones and zeroes of the binary sequence and checks if there is no large difference.

2. Serial Test: The transition characteristics of a sequence such as the number $00,01,10$ and

11 are evaluated. Ideally, it should be uniformly distributed within the sequence.

3. Poker Test: A N length sequence is segmented into blocks of $\mathrm{M}$ bits and the total number of segments is N/M. Within each segment, the integer value can vary from 0 to $\mathrm{m}=2 \mathrm{M}-1$. The objective of this test is to count the frequency of occurrence of each M length segment. Ideally, all the frequency of occurrences should be equal

4. Runs Test: A sequence is divided into contiguous stream of 1's that is referred as blocks and contiguous stream of 0 's that is referred as gaps. If $r_{i 0}$ is the number of gaps of length $i$, then half of the gaps will have length 1 bit, a quarter with length 2 bits, and an eighth with length 3 bits. If ril is the number of blocks of length $i$, then the distribution of blocks is similar to the number of gaps.

After the maximum generation this proposed SA based key generation algorithm will generate best fittest key stream having length of 128 bits.

101100111010000011010100101110110001000010010000111101010000010100001100100010 10000111001111000100000110011110001111100000110101

Now, consider the plain text to be encrypted is "SA Encryption"

01000001/01010011/00100000/01000101/01101110/01100011/01110010/01111001/01110000/0

$1110100 / 01101001 / 01101111 / 01101110$

Here " $/$ " is used as the separator between successive bytes.

In this example plain text size is 104 bits. Here plain text size is less than the size of the 128 bit SA based key stream. So, no need to perform key expansion operation.

Perform XOR operation between plain text and SA based key stream.

So, after the XOR operation cipher text is

111100101111001111110100111111100111111011110011100001110111110001111100111111

10011101011001111001101000 i.e. “òôôp ót+||pužh” 


\section{RESULTS}

Table 2 depicts the average fitness values of different number of generations. Table shows 4 set of entries where 40, 6080,100 number of generations are considered. It is observed from the table that increasing the number of generation also increased the fitness values in average.

Table 2 Average of fitness values

\begin{tabular}{|c|c|}
\hline Number of Generations & Average of fitness values \\
\hline 40 & 35.1486 \\
\hline 60 & 35.8713 \\
\hline 80 & 36.2581 \\
\hline 100 & 36.7316 \\
\hline
\end{tabular}

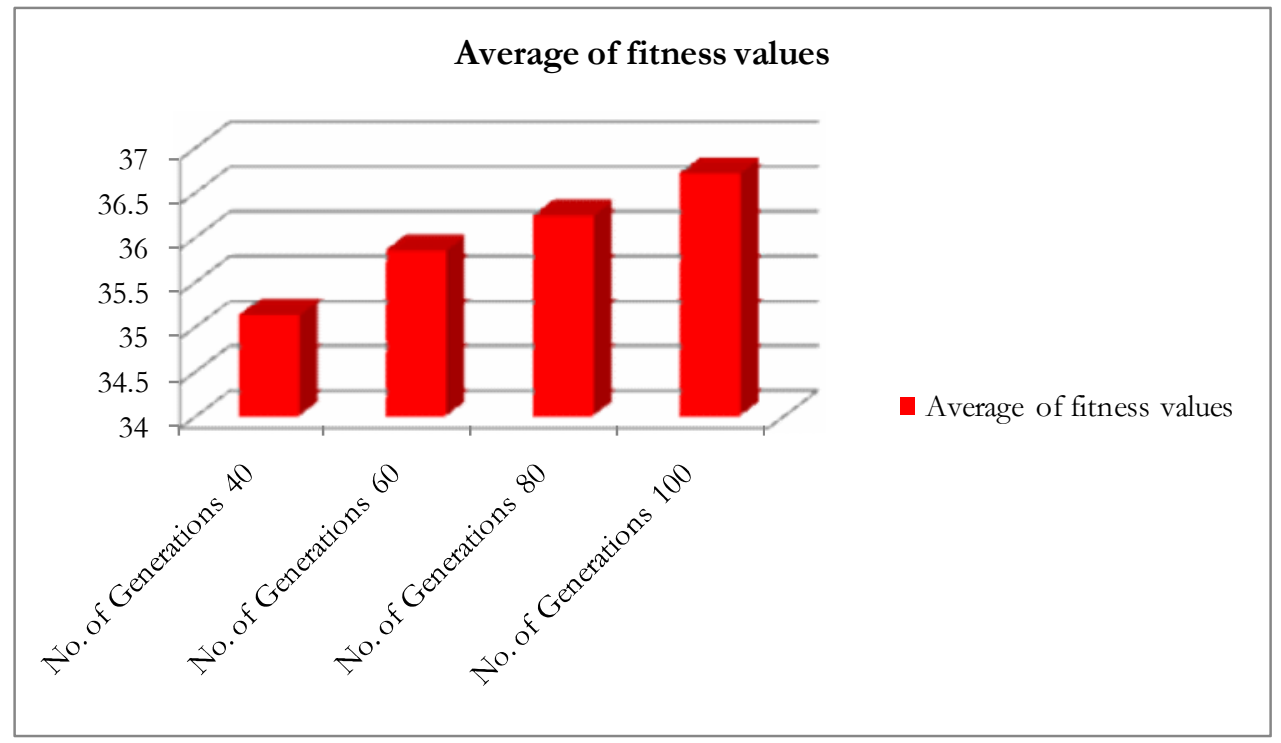

Figure 1 Number of Generation Vs Average of Fitness Values

Table 3 tabulated the fitness values of 50 numbers of iterations and the average fitness value of 50 iterations is 34.89712 
International Journal on Information Theory (IJIT), Vol.2, No.1/2/3/4, October 2013

Table 3 List of fitness values in 50 iterations

\begin{tabular}{|c|c|}
\hline $\begin{array}{c}\text { Iteration } \\
\text { No. }\end{array}$ & $\begin{array}{c}\text { Fitness } \\
\text { Value }\end{array}$ \\
\hline 1 & 31.4377 \\
\hline 2 & 37.5723 \\
\hline 3 & 34.4687 \\
\hline 4 & 36.3263 \\
\hline 5 & 31.8379 \\
\hline 6 & 39.5962 \\
\hline 7 & 41.6294 \\
\hline 8 & 32.6138 \\
\hline 9 & 28.5972 \\
\hline 10 & 32.2379 \\
\hline 11 & 36.7457 \\
\hline 12 & 35.8027 \\
\hline 13 & 28.0429 \\
\hline 14 & 34.7493 \\
\hline 15 & 25.1223 \\
\hline 16 & 43.6024 \\
\hline 17 & 36.9032 \\
\hline 18 & 38.7839 \\
\hline 19 & 27.9125 \\
\hline 20 & 41.5432 \\
\hline 21 & 30.9120 \\
\hline 22 & 35.2396 \\
\hline 23 & 34.9486 \\
\hline 24 & 29.6919 \\
\hline 25 & 27.1037 \\
\hline 26 & 39.6495 \\
\hline 27 & 36.9377 \\
\hline 28 & 38.3426 \\
\hline 16.6485 \\
\hline
\end{tabular}


International Journal on Information Theory (IJIT), Vol.2, No.1/2/3/4, October 2013

\begin{tabular}{|c|c|}
\hline $\begin{array}{c}\text { Iteration } \\
\text { No. }\end{array}$ & $\begin{array}{c}\text { Fitness } \\
\text { Value }\end{array}$ \\
\hline 30 & 32.4891 \\
\hline 31 & 24.8239 \\
\hline 32 & 31.3793 \\
\hline 33 & 36.1682 \\
\hline 34 & 37.8425 \\
\hline 35 & 33.7348 \\
\hline 36 & 42.3876 \\
\hline 37 & 30.9190 \\
\hline 38 & 28.6409 \\
\hline 39 & 40.1002 \\
\hline 40 & 37.6817 \\
\hline 41 & 36.8629 \\
\hline 42 & 38.6328 \\
\hline 43 & 36.1684 \\
\hline 44 & 38.8292 \\
\hline 45 & 32.9716 \\
\hline 46 & 35.4094 \\
\hline 47 & 29.6962 \\
\hline 48 & 42.7356 \\
\hline 49 & 34.8038 \\
\hline 50 & 37.5792 \\
\hline
\end{tabular}


International Journal on Information Theory (IJIT), Vol.2, No.1/2/3/4, October 2013

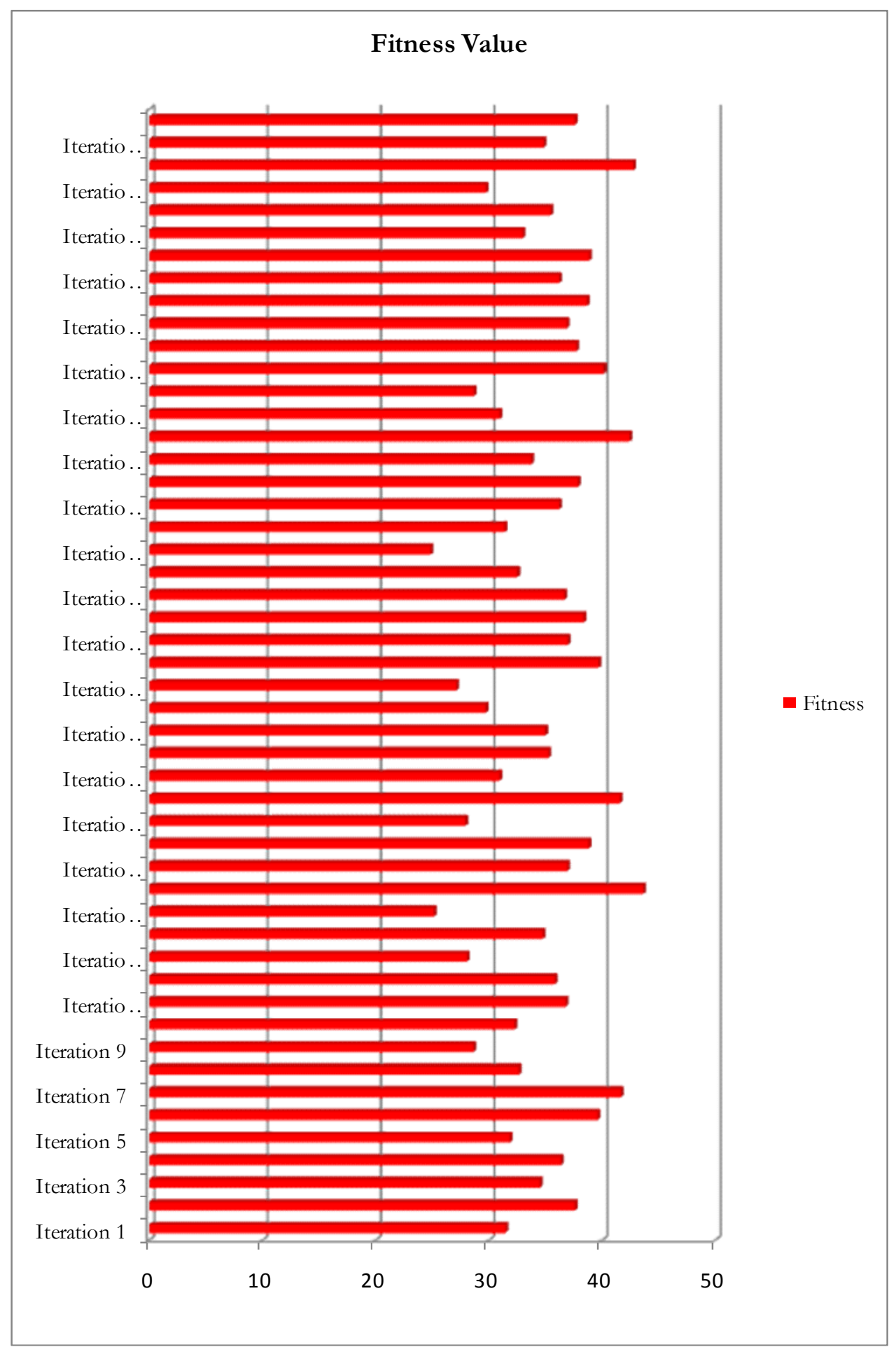

Figure 2 Graph Representation of No. of Iteration Vs Fitness Values

After the maximum generation this proposed SA based key generation algorithm will generate best fittest key stream. First 128 bits from the fittest key stream considered as a SA based key. 
International Journal on Information Theory (IJIT), Vol.2, No.1/2/3/4, October 2013

\section{Key Storage Comparison and Analysis with Existing Methods}

Table 4 shows the comparison of results among Proposed CISAKG, AES, RC4 and Vernam Cipher.

Table 4 Comparison of key storage in Proposed CISAKG, AES, RC4 and Vernam Cipher

\begin{tabular}{|c|c|c|c|c|}
\hline $\begin{array}{c}\text { Length of } \\
\text { Plain text }\end{array}$ & $\begin{array}{c}\text { Key } \\
\text { Storage } \\
\text { Proposed } \\
\text { (CISAKG) }\end{array}$ & $\begin{array}{c}\text { Key } \\
\text { Storage } \\
\text { (AES) }\end{array}$ & $\begin{array}{c}\text { Key } \\
\text { Storage } \\
\text { (RC4) }\end{array}$ & $\begin{array}{c}\text { Key } \\
\text { Storage } \\
\text { (Vernam } \\
\text { Cipher) }\end{array}$ \\
\hline 64 & 128 & 128 & 52 & 60 \\
\hline 120 & 128 & 128 & 106 & 120 \\
\hline 500 & 128 & 128 & 437 & 500 \\
\hline 1000 & 128 & 128 & 913 & 1000 \\
\hline
\end{tabular}

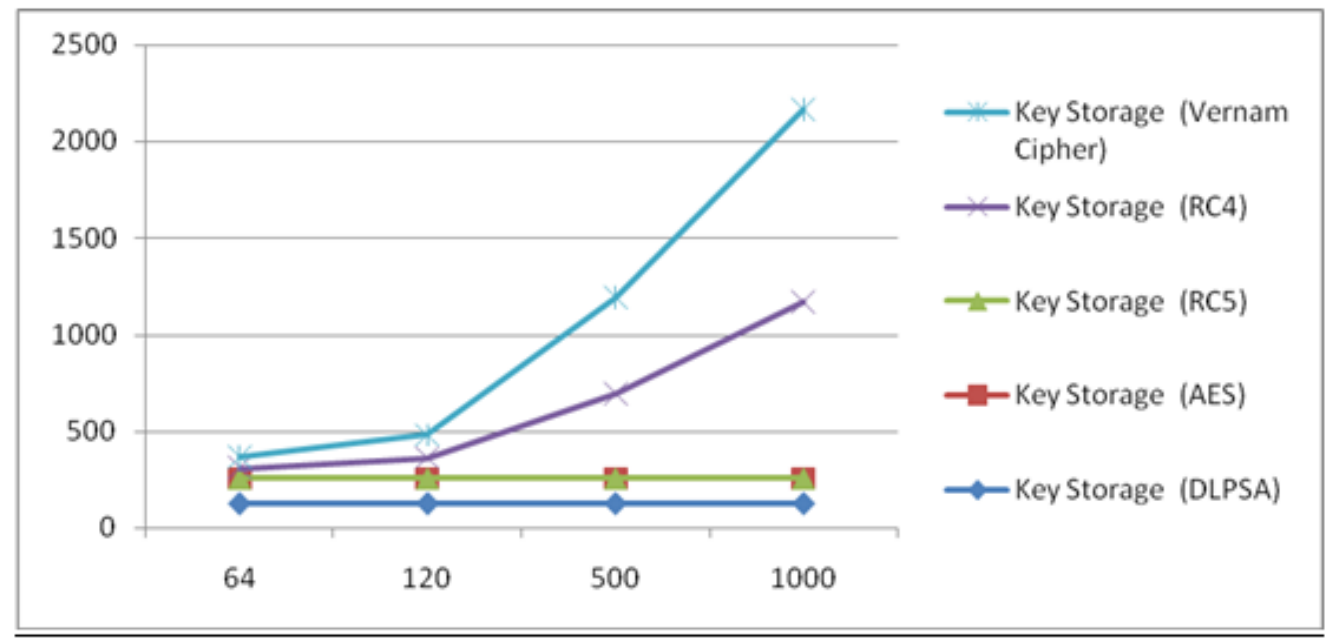

Figure 3 Comparison of key storage in Proposed CISAKG, AES, RC5, RC4 and Vernam Cipher

\section{CONCLUSION}

In CISAKG the number of keys to be stored is at par AES and less when compared to RC4, Vernam Cipher and the keys are generated by passes through a number of statistical tests to examine randomness of the generated key stream, key stream period length, chromosome length using some statistical test like frequency test, serial test, poker test, auto correlation test and runs test. This procedure ensures the robustness of the key. In CISAKG key stream size is 128. If number of bits in a plain text is grater than the key stream then key stream get expanded and if the plain text size is less than 128 bits than the size of the key stream used for encryption is 128 . In AES encryption strategy the minimum key stream requirement is 128 bits. Whereas RC4 stream cipher method is vulnerable to analytic attacks of the state table. 1 out of every 256 keys is a weak key. These keys can be identified by cryptanalysis which can find whether the generated bytes are strongly correlated with the bytes of the key. SA based key generation is a procedure for finding good quality solutions to a large diversity of combinatorial optimization problems. and also helps to avoid from the problem of getting stuck in a local optima In Vernam cipher the keys 
are randomly generated using random stream generator. The drawback is that the number of keys to be stored and distributed should be equal to the length of the plain text. Also the keys used to encrypt the plain text can be found if the random number generator is cracked.

\section{ACKNOWLEDGEMENTS}

The author expresses deep sense of gratitude to the DST, Govt. of India, for financial assistance through INSPIRE Fellowship leading for a PhD work under which this work has been carried out.

\section{REFERENCES}

[1] Mandal, J. K., Sarkar Arindam, “An Adaptive Neural Network Guided Secret Key Based Encryption Through Recursive Positional Modulo-2 Substitution For Online Wireless Communication (ANNRPMS)", in Proc. International Conference on Recent Trends In Information Technology (ICRTIT 2011) Conf. BY IEEE, 3-5 June 2011, Madras Institute of Technology, Anna University, Chennai, Tamil Nadu, India. 978-1-4577-0590-8/11

[2] Mandal, J. K., Sarkar Arindam, "An Adaptive Neural Network Guided Random Block Length Based Cryptosystem (ANNRBLC)", in Proc. $2^{\text {nd }}$ International Conference On Wireless Communications, Vehicular Technology, Information Theory And Aerospace \& Electronic System Technology" (Wireless Vitae 2011) Conf By IEEE Societies, February 28, 2011- March 03, 2011,Chennai, Tamil Nadu, India. ISBN 978-87-92329-61-5.

[3] Mandal, J. K., Sarkar Arindam "Neural Network Guided Secret Key based Encryption through Cascading Chaining of Recursive Positional Substitution of Prime Non-Prime (NNSKECC)" [TP48][PID-63], in Proc. of International Confrence of Computing and Systems-2010 Conf by ICCS-2010, Novembar 19-20, 2010, The University of Burdwan,pp 291-297.

[4] Atul Kahate, Cryptography and Network Security, 2003, Tata McGraw-Hill publishing Company Limited, Eighth reprint 2006.

\section{Arindam Sarkar}

INSPIRE FELLOW (DST, Govt. of India), MCA (VISVA BHARATI, Santiniketan, University First Class First Rank Holder), M.Tech (CSE, K.U, University First Class First Rank Holder). Total number of publications 25.

\section{Jyotsna Kumar Mandal}

M. Tech.(Computer Science, University of Calcutta), Ph.D.(Engg., Jadavpur University) in the field of Data Compression and Error Correction Techniques, Professor in Computer Science and Engineering, University of Kalyani, India. Life Member of Computer Society of India since 1992 and life member of cryptology Research Society of India. Dean Faculty of Engineering, Technology \& Management, working in the field of Network Security, Steganography, Remote Sensing \& GIS Application, Image Processing. 25 years of teaching and research experiences. Eight Scholars awarded Ph.D. and 8 are pursuing. Total number of

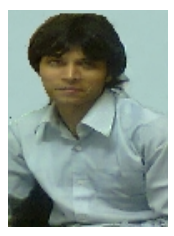
publications 267.

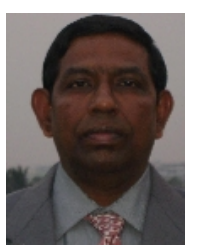

